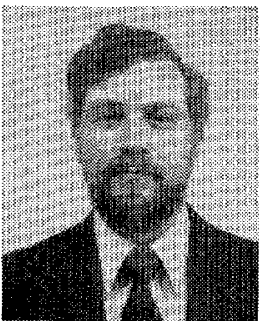

David W. Stowe was born in Three Rivers, MI, on January 1, 1944. He received the B.S. degree in physics from the University of Wisconsin, Madison, in 1966, and the M.S. and Ph.D. degrees in physics from the University of Illinois, Urbana-Champaign, in 1967 and 1971 , respectively.

In 1971 he joined the Applied Physics Laboratory, Johns Hopkins University, Laurel, MD, where he worked on aspects of acoustics and signal processing. In 1977 he joined the Electrical and Electronic Research Division, Gould Laboratories, Rolling Meadows, IL, as Manager of the fiber optics project. In 1981 he was appointed Program Manager of fiber optics, surface acoustic wave devices, and thin film research. His professional interests include fiber optic components and sensors, acoustics, and signal processing.

Dr. Stowe is a member of the American Physical Society, the Optical Society of America, the Acoustical Society of America, and Phi Beta Kappa.

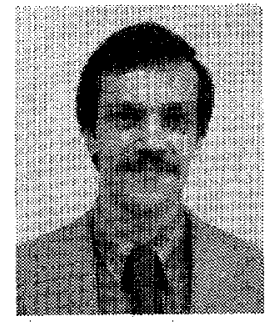

Douglas R. Moore was born in Fort Worth, TX, on June 10,1953. He received the M.S. and Ph.D. degrees in physics from the University of Illinois, Chicago Circle, in 1977 and 1981, respectively.

In 1981 he joined the Electrical and Electronic Research Division, Gould Laboratories, Rolling Meadows, IL, as a Scientist. His areas of interest include fiber optic components, couplers, and sensors.

Dr. Moore is a member of the American Physical Society.

Richard G. Priest, for a photograph and biography, see p. 665 of the April 1982 issue of this Journal.

\title{
Homodyne Demodulation Scheme for Fiber Optic Sensors Using Phase Generated Carrier
}

\author{
ANTHONY DANDRIDGE, ALAN B. TVETEN, AND THOMAS G. GIALLORENZI, SENIOR MEMBER, IEEE
}

\begin{abstract}
A method of homodyne demodulation using a phase generated carrier is described and experimentally demonstrated. The method has a large dynamic range, good linearity, and is capable of detecting phase shifts in the microradian range. The detection scheme obviates the phase tracker resetting problem encountered in active homodyne detection schemes. Two methods of producing the carrier are presented, one employing a piezoelectric stretcher, the other using current induced frequency modulation of the diode laser source. These two methods are compared. The origins of the noise limiting the system are briefly discussed.
\end{abstract}

\section{INTRODUCTION}

$\mathrm{R}$ ECENTLY, there has been considerable interest in using optical fibers as the sensing element in devices such as hydrophones, spectrophones, magnetometers, accelerometers, and ac current sensors [1]. One of the configurations which has shown high sensitivity is that of the Mach-Zehnder allfiber interferometer. In this configuration, there are many methods of detecting relative optical phase shift between the signal and reference fibers. The design of the detection scheme is made nontrivial by the presence of low frequency random temperature and pressure fluctuations which the arms of the interferometer experience. These fluctuations produce

Manuscript received April 1, 1982; revised June 4, 1982.

The authors are with the Naval Research Laboratory, Washington, DC 20375 . differential drifts between the arms of the interferometer. The drift causes changes in the amplitude of the detected signal (signal fading), as well as distortion of the signal (frequency up-conversion).

Several detection schemes are currently available: passive homodyne, active homodyne (phase tracking), true heterodyne, and synthetic heterodyne. Each of these techniques has both advantages and disadvantages. The current state of these detection schemes is reviewed in [1]. At this time, only the active homodyne system has reached a level of high performance $\left(10-10^{-6} \mathrm{rad}\right.$ sensitivity with good linearity and low harmonic distortion), packageability $\left(<24 \mathrm{~cm}^{3}\right)$, and low power consumption. In order to achieve this high level of performance, the technique requires relatively large piezoelectric phase modulators and fast reset circuitry. Large modulators are undesirable in multielement sensors since they increase the active sensor's size and decrease its reliability. Additionally, the need for the sensor circuitry to reset itself every time the environmental noise drives it past its dynamic range adds additional noise. In this paper, a passive homodyne technique which obviates the two problems discussed above is presented. Unlike other passive techniques previously reported [2], this technique has been shown to offer a very high level of performance with a linear dynamic range of $\sim 10^{7}$. This large linear dynamic range allows both small and large amplitude signals commonly encountered in applications to be observed with excellent fidelity. Two methods of utilizing this approach 
(both usable for multisensor systems) are presented as well as the experimental and theoretical limitations of the approaches.

\section{THEORY}

The technique used to detect small signal phase shifts and eliminate signal fading caused by large environmental drifts is achieved by introducing a large amplitude phase shift at a frequency outside of the signal band. These large amplitude signals carry as sidebands the signals of interest. Relatively simple electronic processing schemes have been investigated to remove the desired phase shifts from the large carrier signals [3], [4]. The present approach outlined below offers improvements in terms of detection accuracy and simplicity of electronics.

The variation in the light intensity detected at the output of an interferometer may be written as

$$
I=A+B \cos \theta(t)
$$

where $\theta(t)$ is the phase difference between the arms of the interferometer. The constants $A$ and $B$ are proportional to the input optical power, but $B$ also depends on the mixing efficiency of the interferometer. If a sinusoidal modulation with a frequency $\omega_{o}$ and amplitude $C$ is imposed on the interferometer, then (1) becomes

$$
I=A+B \cos \left(C \cos \omega_{o} t+\phi(t)\right)
$$

where $\phi(t)$ includes not only the signal of interest, but environmental effects as well. Expanding (2) in terms of Bessel functions [5] produces

$$
\begin{aligned}
I= & A+B\left\{\left[J_{0}(C)+2 \sum_{k=1}^{\infty}(-1)^{k} J_{2 k}(C) \cos 2 k \omega_{o} t\right] \cos \phi(t)\right. \\
& \left.-\left[2 \sum_{k=0}^{\infty}(-1)^{k} J_{2 k+1}(C) \cos (2 k+1) \omega_{o} t\right] \sin \phi(t)\right\} .
\end{aligned}
$$

From this expression it is clear that when $\phi(t)=0$, only even multiples of $\omega_{o}$ are present in the output signal, whereas for $\phi(t)=\pi / 2$ rad (quadrature condition), only the odd multiples of $\omega_{0}$ survive.

In a similar fashion the phase angle $\phi(t)$ can be separated into a signal component of frequency $\omega$ and the environmental drifts $\psi(t),(\phi(t)=D \cos \omega t+\psi(t))$ and expanded

$$
\begin{aligned}
\cos \phi(t)= & {\left[J_{0}(D)+2 \sum_{k=1}^{\infty}(-1)^{k} J_{2 k}(D) \cos 2 k \omega t\right] \cos \psi(t) } \\
& -\left[2 \sum_{k=0}^{\infty}(-1)^{k} J_{2 k+1}(D) \cos (2 k+1) \omega t\right] \sin \psi(t) \\
\sin \phi(t)= & {\left[2 \sum_{k=0}^{\infty}(-1)^{k} J_{2 k+1}(D) \cos (2 k+1) \omega t\right] \cos \psi(t) } \\
& +\left[J_{0}(D)+2 \sum_{k=1}^{\infty}(-1)^{k} J_{2 k}(D) \cos 2 k \omega t\right] \sin \psi(t) .
\end{aligned}
$$

These equations along with (3) show that when $\psi(t)=0$, even (odd) multiples of $\omega$ are present in the output signal centered about the even (odd) multiples of $\omega_{o}$. For the case when $\psi(t)=\pi / 2 \mathrm{rad}$, even (odd) multiples of $\omega$ are present about the odd (even) multiples of $\omega_{o}$. This behavior is shown in Fig. 1(a) and (b) for the sidebands produced by $\omega$ about 0 , $\omega_{o}$, and $2 \omega_{o}$.

The sidebands contain the signal of interest and are either present about the even or the odd multiples of $\omega_{o}$. The signal is obtained by mixing the total output signal with the proper multiple of $\omega_{o}$ and low-pass filtering to remove the terms above the highest frequency of interest.

For the carrier frequencies considered in the experiment, namely $0, \omega_{o}$, and $2 \omega_{o}$, the output signals after mixing and filtering are

$$
\begin{aligned}
& A+B J_{0}(C) \cos \phi(t) \\
& B G J_{1}(C) \sin \phi(t) \\
& -B H J_{2}(C) \cos \phi(t),
\end{aligned}
$$

respectively, and where $G$ and $H$ are the amplitude of the mixing signals for $\omega_{o}$ and $2 \omega_{o}$.

In order to obtain a signal that does not fade as a function of undesired fluctuations, two signals, one containing the sine $\phi(t)$ and the other cosine $\phi(t)$ are utilized. The time derivative of the sine and cosine terms are cross multiplied with the cosine and sine terms, respectively, to yield the desired sine and cosine squared terms [1]. The process will be illustrated by considering the output signals for $\omega_{o}$ and $2 \omega_{o}$. The time derivative of these are obtained from (5) and are given by

$$
\begin{aligned}
& B G J_{1}(C) \dot{\phi}(t) \cos \phi(t) \\
& B H J_{2}(C) \dot{\phi}(t) \sin \phi(t) .
\end{aligned}
$$

Multiplying this by the signal for the other frequency produces

$$
B^{2} G H J_{1}(C) J_{2}(C) \dot{\phi}(t) \cos ^{2} \phi(t)
$$

and

$$
-B^{2} G H J_{1}(C) J_{2}(C) \dot{\phi}(t) \sin ^{2} \phi(t) .
$$

Subtracting gives

$$
\begin{aligned}
& B^{2} G H J_{1}(C) J_{2}(C) \dot{\phi}(t)\left(\sin ^{2} \phi(t)+\cos ^{2} \phi(t)\right) \\
& =B^{2} G H J_{1}(C) J_{2}(C) \dot{\phi}(t) .
\end{aligned}
$$

This output can then be integrated to produce the signal $\phi(t)$ which includes all of the drift information in addition to the actual signal.

A similar set of equations could be written for the 0 and $\omega_{0}$. However, in this case a stable offset must be introduced to remove the dc term $A$ from the output. If this term is not removed, the output signal $\dot{\phi}(t)$ will depend on the value of $\phi(t)$.

The actual value of the coefficients in (8) are not important. The value of the argument in the Bessel functions should be chosen so that $J_{1}(C)$ and $J_{2}(C)$ are equal to reduce the dependence on slight variations in $C$. To maximize the signal-to- 


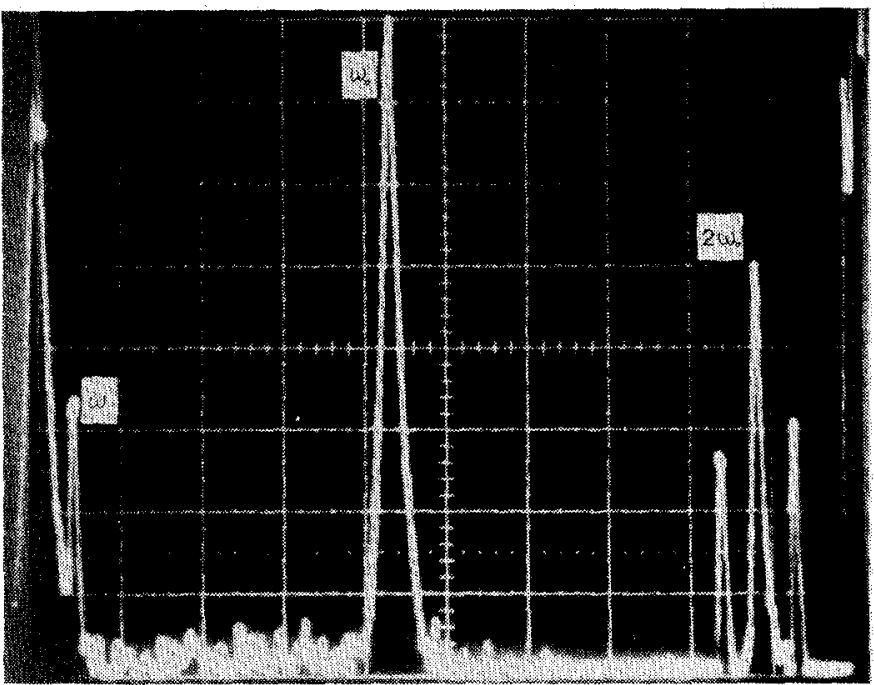

(a)

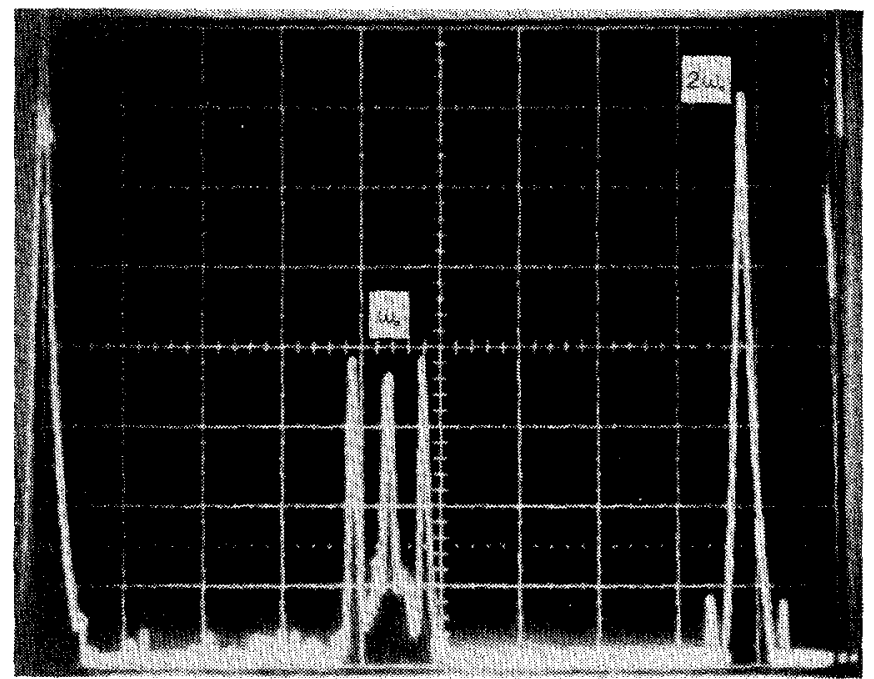

(b)

Fig. 1. Spectrum analyzer trace of the output of the interferometer driven with a large amplitude signal at $\omega_{0}$ and small signal at $\omega$. (a) In quadrature $\psi(t)=\pi / 2 \mathrm{rad}$. (b) Out of quadrature $\psi(t)=0$. Vertical scale is linear, horizontal scale is $2 \mathrm{kHz} /$ div.

noise ratio, the value of the amplitudes $G$ and $H$ should be as large as possible without overloading the electronics. The factor $B$ depends on the optical power and the mixing efficiency in the interferometer and is the most difficult to control. Any variation in the output of the beamsplitters or a polarization change in the interferometer arms could induce a change in $B$. A solution to this is to put in an automatic gain control in the circuit to ensure a fixed modulation amplitude.

\section{EXPERIMENT}

As discussed in the previous section, the principle of operation of this demodulation scheme relies on the fact that, if the interferometer output is composed of two terms which are proportional to sine and cosine, these terms may be processed to eliminate signal fading. In the first part of this section, the production of the cosine and sine terms will be discussed; in the second part, the electronic processing will be presented.
The experimental configuration employed to demonstrate this homodyne scheme was that of a bulk optic Michelson interferometer. This system (described in detail elsewhere) [6] allowed accurate control of the optical path difference and could readily be isolated from environmental noise sources in our setup. Typically, the interferometer noise of this system was $2 \times 10^{-7} \mathrm{rad}$ (at $1 \mathrm{kHz}$ ). Both mirrors of the Michelson interferometer were mounted on piezoelectric cylinders so that both "real" and drift signals could be produced. The bulk Michelson interferometer was chosen rather than the fiber system to allow greater flexibility in control of the optical path difference. The fact that a bulk interferometer was used is not essential to determining the usefulness of this technique since all-fiber interferometers (powered by diode lasers) of sub microradian performance have been built and operated routinely [1]. Consequently, the results of this work are directly applicable to these systems. In this experiment a Hitachi HLP 1400 laser was used as the source.

The implementation of the demodulation scheme requires that a high frequency carrier signal be produced in the interferometer. The following two methods were employed: 1) piezoelectric cylinder, and 2) modulating the emission frequency of the laser diode by modulating the laser drive current. The first method has the advantage that a zero optical path difference may be used in the interferometer. The second method has the advantage of eliminating the piezoelectric cylinder as well as allowing the interferometer part of the fiber system to be removed from electrical components. This scheme has the disadvantage that to convert the current induced frequency shift to a relative phase shift, a nonzero optical path difference is required, thus the phase noise [6] can become the factor limiting the sensitivity of the sensor. This limitation will be discussed in detail in the experimental performance section.

A carrier wave phase shift of the desired amplitude (i.e., 1.4 or $2.2 \mathrm{rad}$ ) was easily obtained using the piezoelectric cylinder at the fundamental and first harmonic of its longitudinal resonance which occured at 50 and $100 \mathrm{kHz}$, respectively. To produce the cosine and sine terms, the circuit shown in Fig. 2 was used. For the case illustrated in Fig. 2(a) the fundamental of the signal and the sideband of the fundamental of the carrier were used, whereas the sidebands of the fundamental of the carrier and the first harmonic of the carrier were utilized in the implementation shown in Fig. 2(b). The use of the two configurations will be discussed in greater detail later.

The production of the carrier using the modulation of the diode lasers driving current is dependent on the optical path difference and is additionally a function of the modulation frequency [7]. For the particular laser used, the decrease in the modulation index with modulation frequency is shown in Fig. 3. As can be seen, the modulation index $d v / d i$ has only decreased by $\sim 0.75$ from its dc value $\left((d v / d i)_{o}\right)$ at a modulation frequency of $100 \mathrm{kHz}$. This shows that this technique can be used efficiently at least up to this frequency for this particular laser. It should be noted that for many lasers, $(d v / d i)$ has decreased to $\sim 0.2$ of the dc value at $100 \mathrm{kHz}$ and are thus unsuitable for the use in this application [7]. It 
(a)

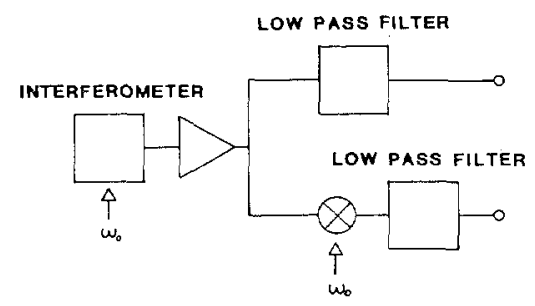

(b)

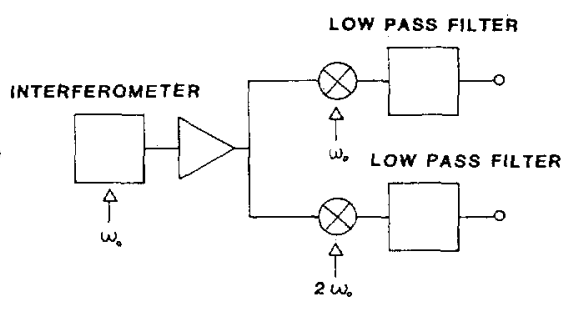

Fig. 2. Circuit used to produce sine and cosine of the phase shift: (a) using the 0 and $\omega_{0}$ frequency carrier; (b) using the $\omega_{0}$ and $2 \omega_{0}$ frequency carrier.

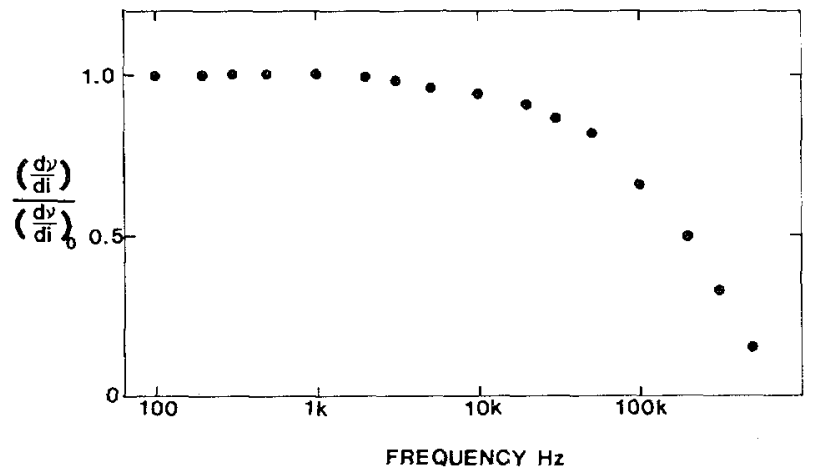

Fig. 3. Frequency response of the diode laser modulation index $(d v / d i)$ compared to the dc value $(d \nu / d i)_{o}$ for the Hitachi HLP 1400 laser used in the experiment.

should also be noted that the use of laser current modulation to produce the carrier may also be used in the synthetic heterodyne approach described by Cole et al. [3]. The use of this technique eliminates the use of the piezoelectric cylinder in that scheme, but because of the reported poor sensitivity of the synthetic heterodyne approach, no effort was made to construct this system.

Fig. 1(a) and (b) shows the output of the interferometer; an $8.8 \mathrm{kHz}$ carrier, produced by modulation of the laser diode, and an $0.01 \mathrm{rad}$ signal (produced using a piezoelectric cylinder) at $450 \mathrm{~Hz}$ have been applied to the interferometer. An intermediate value of the amplitude of the carrier signal was used so that the operation in both the configuration of Fig. 2(a) and (b) may be seen. Fig. 1(a) shows the output of the interferometer when the interferometer is in quadrature. Here the fundamental of the carrier and the signal, and the sideband of the first harmonic of the carrier appear strongly. Fig. 1(b) shows the output when the interferometer is out of quadrature. Here the sideband of the fundamental of the carrier and the first harmonic of the carrier appear strongly, whereas the amplitude of the output at the fundamental of the signal and carrier frequency, as well as the sideband of the first harmonic of the carrier, appear only weakly. It can be seen that the sine and cosine terms required can be extracted from either the signal fundamental and the sidebands of the fundamental of the carrier [Fig. 2(a)] or the sideband of the fundamental and first harmonic of the carrier [Fig. 2(b)]. As has been discussed in the theory section, the amplitudes of the sine and cosine terms are determined by the depth of modulation of the carrier. An example of the output after processing using the configuration of Fig. 2(a) and adjustment of the carrier amplitude to $1.4 \mathrm{rad}$ is shown in Fig. 4. Here the Lissajou figure derived from the two outputs (sine and cosine) have been displayed by using them as the $x$ and $y$ inputs of an oscilloscope. With no signal applied, the output appears as a dot which describes a circle as the interferometer and drifts into and out of quadrature. To show the circle (indicating that the amplitudes of the sine and cosine terms are equal) more clearly, a low frequency $(\sim 100 \mathrm{~Hz})$ signal with an amplitude of $\pm \pi \mathrm{rad}$ has been applied to the interferometer.

A similar result to that shown in Fig. 4 was achieved using the configuration shown in Fig. 2(b), but here an amplitude of the carrier close to the predicted $2.2 \mathrm{rad}$ was required for circularity. It should be mentioned that use of the double sideband technique [Fig. 2(b)] conveys the advantage that both outputs from the interferometer may be ac coupled which eliminates a number of problems associated with dc drifts. Obviously, in the configuration used in Fig. 2(a) the signal obtained from the fundamental of the signal frequency needs to be de coupled.

To obtain the signal from the output of Fig. 2 (i.e., the sine and cosine terms) the differentiate and cross multiply technique described in the theory section was employed. A schematic of the circuit is shown in Fig. 5, the multipliers were Analog Devices model $435 \mathrm{~K}$ while the differentiators, difference amplifier, and integrator were made from low cost op amps.

\section{Performance of System}

This section is divided into two parts; part 1) concerns the operation of the system where the two methods of production of the carrier gave identical results and part 2) concerns the minimum detectable phase shift (sensitivity) which was a function of the carrier production technique.

1) The signal processing technique was tested to evaluate how well the inherent fading problem of the homodyne interferometer was overcome. To do this, a $0.01 \mathrm{rad}(\mathrm{rms})$ signal at frequency $f_{s}$ was applied to one arm of the interferometer while a slow $(0.05 \mathrm{~Hz})$ ramp of $\sim 10 \pi \mathrm{rad}$ was applied to the other arm. The slow ramp had the effect of successively changing the relative phase shift in the interferometer such that all relative phase angles were encountered. The amplitude of the output of the integrator at $f_{s}$ was found to be a constant to within $\sim 5$ percent. The small amplitude variation of the output was attributed to a slight imbalance of the electronics. The signal output also remained constant when the drift frequency was increased to $\sim 5 \mathrm{~Hz}$, which corresponded to a drift of $\sim 150 \mathrm{rad} / \mathrm{s}$. Above this drift rate, although the amplitude of the signal output remained approximately constant, a degradation in the signal-to-noise ratio of the output was observed. 


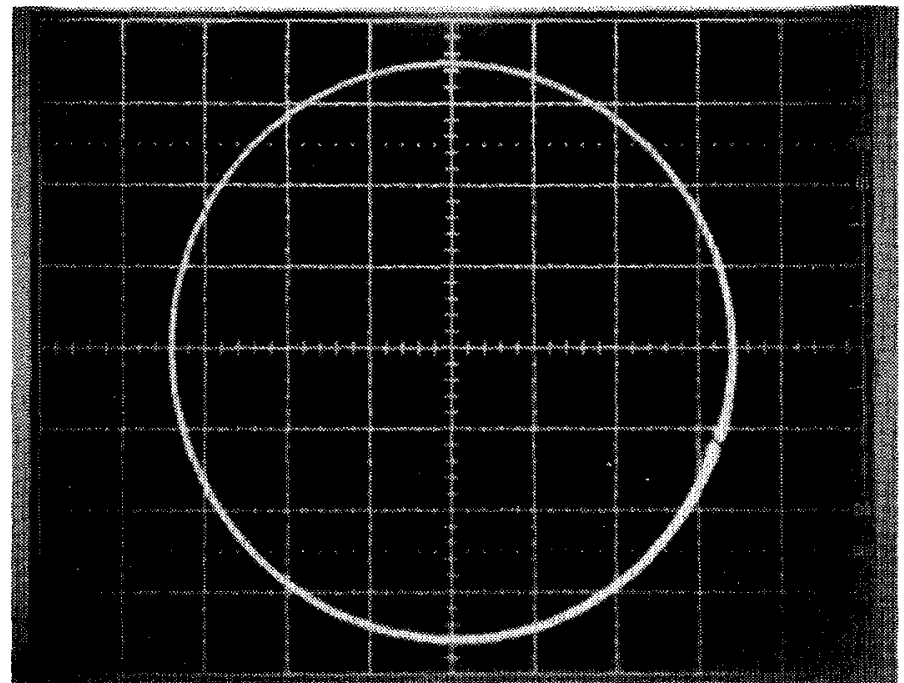

Fig. 4. The sine and cosine outputs of the phase shift using the circuit shown in Fig. 1(a). The two Bessel functions $J_{0}(C)$ and $J_{1}(C)$ are made equal $(C=1.4 \mathrm{rad})$.

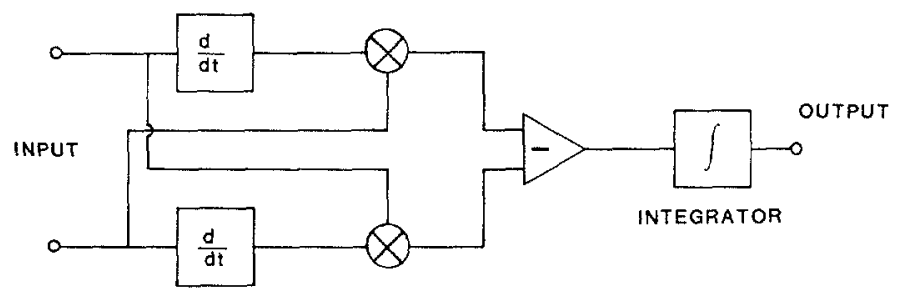

Fig. 5. The schematic of the circuit used to demodulate the sine and cosine of the phase shift.

The frequency response (to the signal frequency $f_{s}$ ) of the system is shown in Fig. 6. The decrease in response at high frequency is due to the low-pass filtering shown in Fig. 2. The frequency response can be extended to higher frequencies if desired by using higher order filters (a simple second order filter was used in this experiment). The rolloff at low frequency was due to the presence of a bleeder resistor across the integrator whose value could be increased if a flat response below $\sim 20 \mathrm{~Hz}$ is required. The system responded linearly to the amplitude of the input signal between the noise floor of the configuration used (typically $10^{-5}-10^{-6} \mathrm{rad}$ ) and $\sim 1 \mathrm{rad}$. Above this signal level ( $\sim 1 \mathrm{rad})$, distortions were observed due to the differentiators overloading from the presence of the residual carrier signal, thus the dynamic range of the system at high signal levels may be increased by 1) lowering the gain prior to the differentiations or preferably, 2) having a sharper cutoff of the carrier modulation (i.e., a higher order filter). Below this region of obvious distortion, the first harmonic of the signal was typically buried in the noise floor and hence was unobservable, but the ratio of the fundamental to the first harmonic was typically greater than $70 \mathrm{~dB}\left(\right.$ i.e., $3 \times 10^{3}$ ).

2) As discussed earlier, the operation of the current induced modulated carrier requires the interferometer to use a nonzero path length difference such that the frequency modulator is converted to the required phase modulation. Consequently, this nonzero path difference allows the phase noise to contribute to the interferometer noise. Using the diode laser modulation scheme, the frequency dependence of the mini-

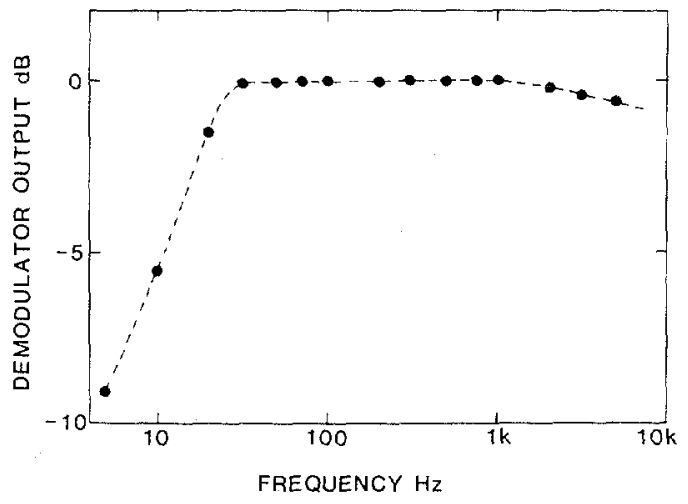

Fig. 6. Frequency response of the demodulator system.

mum detectable phase shift was determined for two path length differences, 10 and $2 \mathrm{~cm}$. The result for the $10 \mathrm{~cm}$ path difference is indicated on the spectrum analyzer trace shown in Fig. 7, where a $0.01 \mathrm{rad}$ signal has been applied to the interferometer at $470 \mathrm{~Hz}$. The noise floor $\left(\sim 3 \times 10^{-5} \mathrm{rad}\right.$ at $1 \mathrm{kHz}$ ) is thought to be due to the phase noise because when the interferometer was operated in a conventional homodyne mode (in quadrature) a noise floor similar to that in Fig. 7 was observed. This experiment was repeated with a 2 $\mathrm{cm}$ path difference, and the noise dropped by approximately a factor of 5 . To eliminate the effect of the phase noise, the same experiment was repeated at zero path length difference. However, here the modulation for the carrier used the mirror mounted on a piezoelectric cylinder. The results for this configuration are shown in Fig. 8. Again, a 0.01 rad signal was applied to the interferometer. The noise floor corresponds to $\sim 10^{-6}$ rad at $1 \mathrm{kHz}$. Above $600 \mathrm{~Hz}$, a number of spurious electronic noise peaks related to the line frequency were observed above the true noise floor.

The results of the three experiments performed above are summarized in Fig. 9, where the solid line in the upper two curves represent the average level of the phase noise. The lowest curve was obtained for the interferometer held manually in quadrature using the conventional homodyne detection scheme. As can be seen, the passive homodyne result is approximately a factor of 2 worse than the result obtained with the conventional homodyne. The noise in the conventional homodyne system was determined by the intrinsic intensity noise of the diode laser [8]. Owing to the carrier modulation, the average level of the intensity noise remains the same as with the conventional homodyne interferometer field in quadrature, however, the amplitude of the phase signal is reduced by the magnitude of the relevant Bessel function (5). Consequently, in an interferometer dominated by the laser intensity noise, a factor of $\sim 2$ difference between the two detection methods is expected for this modulation amplitude.

In an unbalanced interferometer powered by a laser diode, the primary noise source becomes the phase noise due to the fluctuation of the emission frequency of the laser. For a signal-to-noise ratio of 1 , the minimum detectable phase shift $\psi_{m}$ in the interferometer is given by

$$
\psi_{m}=2 \pi D\left(\frac{\delta \nu_{n}}{c}\right)
$$




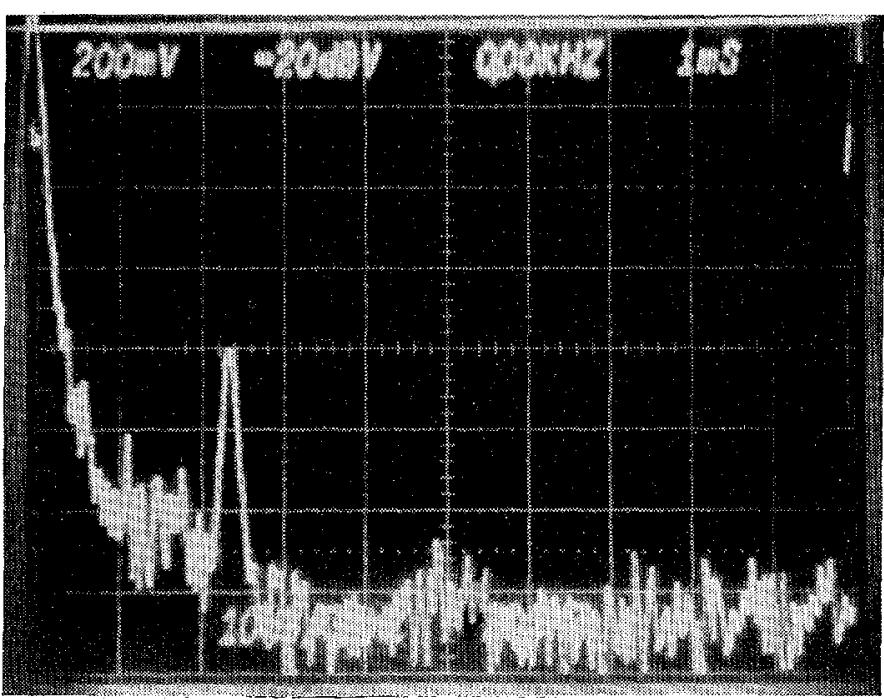

Fig. 7. Spectrum analyzer trace for a $0.01 \mathrm{rad}$ signal at $470 \mathrm{~Hz}$ and the noise floor for a $30 \mathrm{~Hz}$ bandwidth. The vertical scale is $10 \mathrm{~dB} / \mathrm{div}$, horizontal is $200 \mathrm{~Hz} /$ div. The interferometer path length difference is $10 \mathrm{~cm}$. Laser modulation was used to produce the carrier.

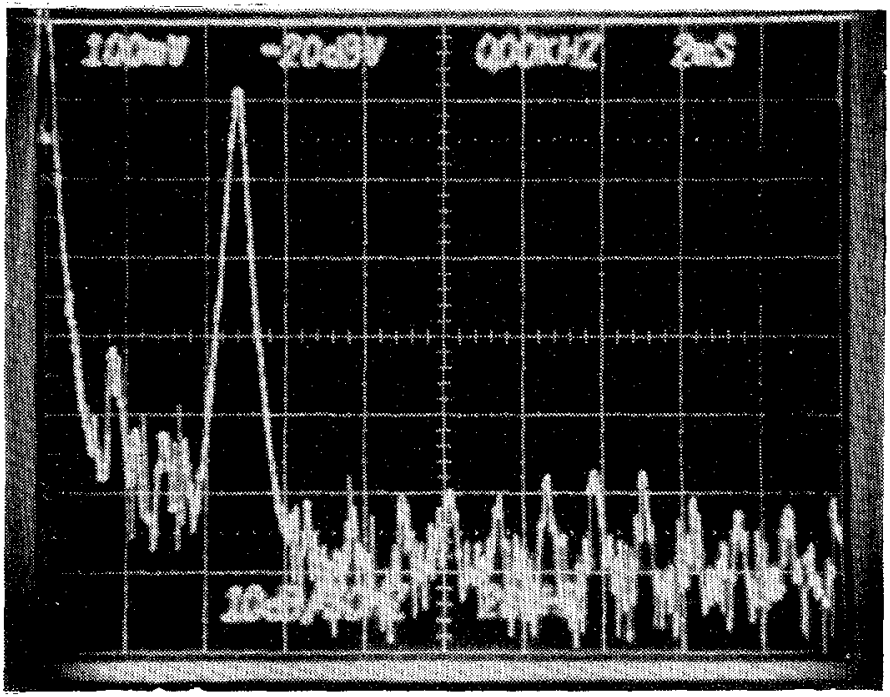

Fig. 8. Spectrum analyzer trace for a $0.01 \mathrm{rad}$ signal at $470 \mathrm{~Hz}$ and the noise floor for a $30 \mathrm{~Hz}$ bandwidth. The vertical scale is $10 \mathrm{~dB} / \mathrm{div}$, horizontal is $200 \mathrm{~Hz} /$ div. The interferometer path length difference is set to zero. Piezoelectric modulation was used to produce the carrier.

where $D$ is the optical path difference, $c$ is the speed of light in free space, and $\delta v_{n}$ is the rms frequency instability of the laser source. Thus, for low noise of operation, $D$ should be made as small as possible. However, to produce the $2.2 \mathrm{rad}$ phase shift at $100 \mathrm{kHz}$ to operate the detection scheme using the configuration of Fig. 1(b), it is necessary to operate the interferometer with a nonzero path difference. The relationship between the frequency shift and the phase shift is also given by

$$
\phi=2 \pi D\left(\frac{\delta \nu}{c}\right)
$$

thus for a pk-pk phase excursion of $4 \cdot 4 \mathrm{rad}, D \delta \nu=0.21$ $\mathrm{GHz} \cdot \mathrm{m}$. For the HLP 1400 used for this study $(d v / d i)_{o} \approx 3$ $\mathrm{GHz} / \mathrm{mA}$ and $\mathrm{pk}-\mathrm{pk}$ value of $3 \mathrm{~mA}$ is assumed (the laser

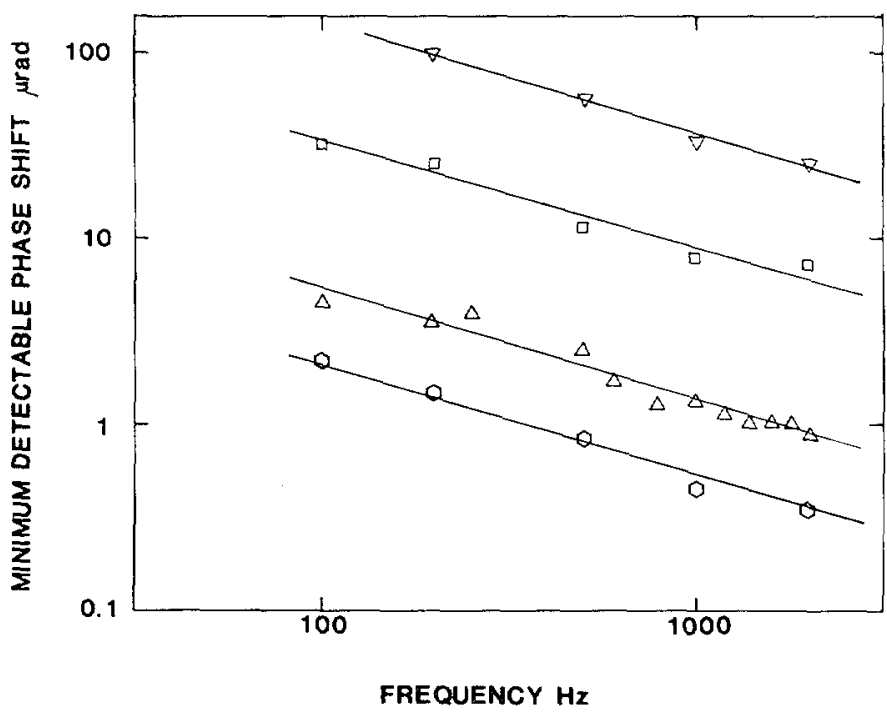

Fig. 9. The minimum detectable phase shift as a function of frequency for $O$ : conventional homodyne scheme manually held in quadrature; $\triangle$ : this passive homodyne scheme with zero optical path length difference; $\square$ : this passive homodyne scheme with $2 \mathrm{~cm}$ optical path length difference; $\nabla:$ this passive homodyne scheme with $10 \mathrm{~cm}$ optical path length difference.

remains in a single mode over this current excursion), then a value for $D$ of $\sim 2.3 \mathrm{~cm}$ is calculated. Thus, the performance of the diode modulation scheme operated with $2 \mathrm{~cm}$ path difference is close to the optimum performance of this detection scheme.

\section{CONCLUSION}

It has been shown that the homodyne detection scheme using a phase generated carrier described is capable of high sensitivity $\left(10^{-6} \mathrm{rad}\right.$ detectability at $\left.1 \mathrm{kHz}\right)$, high dynamic range, and good linearity. It has also been shown that this scheme can operate using a current induced frequency modulation to produce the phase carrier, thus completely isolating the interferometer from electrical components. In this configuration, a $2 \mathrm{~cm}$ path difference is necessary, limiting sensitivity to $9 \times 10^{-6} \mathrm{rad} 1 \mathrm{kHz}$.

\section{ACKNOWLEDGMENT}

The authors would like to thank R. O. Miles for helpful discussions regarding the modulation of the diode laser. Two of the authors (A. Dandridge and A. B. Tveten) wish to thank J. H. Cole who had independently suggested that this method of demodulation could be an alternative to synthetic heterodyne approaches.

\section{REFERENCES}

[1] T. G. Giallorenzi, J. A. Bucaro, A. Dandridge, G. H. Sigel, J. H. Cole, S. C. Rashleigh, and R. G. Priest, "Optical fiber sensor technology," IEEE J. Quantum Electron., vol. QE-18, pp. 626666, Apr. 1982.

[2] S. K. Sheem, T. G. Giallorenzi, and K. P. Koo, "Optical techniques to solve the signal fading problem in fiber interferometers," $A p p l$. Opt., vol. 21, pp. 689-694, 1982; and A. Olsson and C. L. Tang, "Dynamic interferometry techniques for optical pathlength measurements," Appl. Opt., vol. 20, pp. 3503-3507, 1981.

[3] J. H. Cole, B. A. Danver, and J. A. Bucaro, "Synthetic heterodyne interferometric demodulation," IEEE J. Quantum Electron., vol. QE-18, pp. 694-697, Apr. 1982. 
[4] E. L. Green and P. G. Cable, "Passive demodulation of optical interferometric sensors," IEEE J. Quantum Electron., this issue, pp. 1639-1644.

[5] M. Abramowitz and I. A. Stegum, Handbook of Mathematical Functions. Washington, DC: Nat. Bur. Standards.

[6] A. Dandridge, A. B. Tveten, R. O. Miles, D. A. Jackson, and T. G. Giallorenzi, "Single-mode diode laser phase noise," Appl. Phys. Lett., vol. 37, pp. 77-78, 1981.

[7] A. Dandridge and L. Goldberg, "Current induced frequency modulation in diode lasers," Electron. Lett., vol. 18, pp. 302-303, 1982.

[8] A. Dandridge, A. B. Tveten, R. O. Miles, and T. G. Giallorenzi, "Laser noise in fiber-optic interferometer systems," Appl. Phys. Lett., vol. 37, pp. 526-527, 1980.

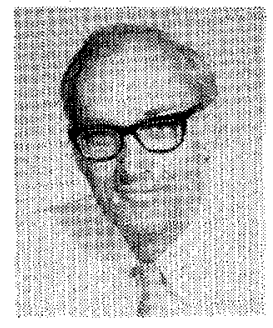

Alan B. Treten was born in Wolf Point, MT, on January 24,1934 . He received the B.A. degree from Concordia College, Moorhead, MN, the M.A. degree from the University of Nebraska, Lincoln, and the Ph.D. degree from Colorado State University, Fort Collins.

Before joining the Naval Research Laboratory, Washington, DC, in 1979, he worked as a Physics Teacher at Dana College, Mankato State University, Mankato, $\mathrm{MN}$, and Colorado State University. He has done research in secondary electron emission in metals, magnetic susceptibility of rare earth oxides, and remote sensing using light scattering from aerosols. Since 1979 he has been working with the fiber optic sensor and the optical information processing groups at NRL.
Anthony Dandridge, for a photograph and biography, see p. 564 of the April 1982 issue of this Journal.
Thomas G. Giallorenzi (SM'78), for a photograph and biography, see p. 458 of the April 1982 issue of this JouRnal.

\title{
Recent Developments in Monolithic Integration of InGaAsP/InP Optoelectronic Devices
}

\author{
UZIEL KOREN, SHLOMO MARGALIT, T. R. CHEN, K. L. YU, AMNON YARIV, FELLOW, IEEE, \\ NADAV BAR-CHAIM, KAM Y. LAU, MEMBER, IEEE, AND ISRAEL URY
}

(Invited Paper)

\begin{abstract}
Monolithically integrated optoelectronic circuits combine optical devices such as light sources (injection lasers and light emitting diodes) and optical detectors with solid-state semiconductor devices such as field effect transistors, bipolar transistors, and others on a single semiconductor crystal. Here we review some of the integrated circuits that have been realized and discuss the laser structures suited for integration with emphasis on the InGaAsP/InP material system. Some results of high frequency modulation and performance of integrated devices are discussed.
\end{abstract}

\section{INTRODUCTION}

$\mathrm{T}$ $\mathrm{HE}$ monolithic integration of optoelectronic devices composed of the III-V compounds GaAlAs/GaAs and InGaAsP/InP has been a topic of considerable interest in recent years as materials and device technology has advanced. In particular, the quaternary InP/InGaAsP material system has

Manuscript received April 1, 1982; revised June 4, 1982. This work was supported in part by the Office of Naval Research and the National Science Foundation (Optical Communication Program).

U. Koren, T. R. Chen, K. L. Yu, and A. Yariv are with the California Institute of Technology, Pasadena, CA 91125.

S. Margalit was on leave at the California Institute of Technology, Pasadena, CA 91125 . He is with the Department of Electrical Engineering, Technion-Israel Institute of Technology, Haifa, Israel.

N. Bar-Chaim, K. Y. Lau, and I. Ury are with Ortel Corporation, Alhambra, CA 91803. been a subject of intense study since devices made of these materials operate in the 1.1-1.6 $\mu \mathrm{m}$ spectral region, which coincides with the optimal region for fiber optics communication systems. As progress is being made in fiber optic technology and low loss single-mode fibers become available, there is also a strong interest in developing semiconductor devices capable of launching, receiving, and processing optical signals at the extremely high data rates that are possible with fiber optic transmission systems. The monolithic integration of optoelectronic devices on a single semiconductor crystal [1], [2] offers the potential advantages of improved reliability, low cost, and small size and also makes possible very fast operation by reducing the circuit parasitic reactances.

The most promising light source for optoelectronic devices is the semiconductor laser diode. This is due to the relatively high power that lasers can launch into fibers, and also because lasers can be modulated at very high frequencies. The lasers that have been studied for integration purposes are sometimes fabricated on semi-insulating (SI) substrates such as $\mathrm{Fe}$ doped InP or Cr doped GaAs. These crystals have very high specific resistivities which can be of the order of $10^{8} \Omega \cdot \mathrm{cm}$ for $\mathrm{Fe}$ doped InP. Although several optoelectronic devices have been integrated on conductive substrates (some will be mentioned in Sections III and IV), the SI substrates offer some important advantages. The advantages of the use of SI sub- 